\title{
On the Transmission Capacity of Wireless Multi-Channel Ad Hoc Networks with local FDMA scheduling
}

\author{
Jens P. Elsner, Ralph Tanbourgi and Friedrich K. Jondral \\ Karlsruhe Institute of Technology, Germany \{jens.elsner, ralph.tanbourgi, friedrich.jondral $\} @$ kit.edu
}

\begin{abstract}
The problem of the capacity of multi-channel interference-limited ad hoc networks with local frequency planning is examined in the transmission capacity framework. Bounds on outage and transmission capacity are given. If local frequency planning, i.e., frequency domain orthogonalization of neighbors in the communication range, is employed, transmission capacity can be significantly improved. The gain on the transmission capacity depends on the node density and is found to be a factor of 1.35 to 13 for a path loss attenuation $\alpha=4$ and outage probability greater than 0.01 . A design insight is, that, if frequency planning in the communication range of nodes is employed, low node densities call for lower spectral efficiencies, while at high node densities higher spectral efficiencies increase the capacity of the network.
\end{abstract}

\section{INTRODUCTION}

The study of dense, interference-limited wireless networks is a topic of recent research interest. From an information theoretic standpoint, it only makes sense to use high or even infinite bandwidth for data transmission over a channel in the absence of interference. Interference, i.e., the need to co-exist with other transmitters, is the fundamental reason to limit system bandwidths. Various technical difficulties also complicate the design of very broadband wireless systems: due to issues such as necessary dynamic range, power consumption or co-site interference, in implementations of wireless networks the total available tuning bandwidth is often much greater than the high frequency bandwidth of the physical layer. A practical example is military communications in the $\mathrm{VHF} / \mathrm{UHF}$ band, where point-to-point data rates of several MBit/s have to be supported in a bandwidth of a few hundred MHz. Hence, it makes sense to evaluate the capacity of wireless ad hoc networks with large operating but limited system bandwidth. An obvious question to answer is that of how to choose the system bandwidth in relation to the operating bandwidth from an information theoretic viewpoint and how many nodes can be supported in the network. We will try to answer this question in the recently popularized transmission capacity framework [1].

\section{Related Work}

Weber et al. compare FH-CDMA and DSSS-CDMA systems under a power constraint with equivalent total occupied bandwidth [2], [3]. They find that FH-CDMA systems have an advantage in terms of transmission capacity.
Jindal et. al. answer the question on how to split a given operating bandwidth to maximize spectral area efficiency [4].

Weber et. al [5] derive the transmission capacity for a network employing interference cancelation. In contrast to this work, we consider the bandwidth split under the assumption that the network locally coordinates FDMA channels. Interference is hence not canceled, but avoided, which makes sense in the light of the findings in [2], [3], [6].

\section{Contribution}

We build on the cited results by Weber, Jindal et al. to show the principal limits of frequency agile networks with an FDMA MAC capable of locally organizing channels in terms of transmission capacity metric in the interferencelimited regime (neglecting thermal noise). A contribution is the application of Brooks' theorem [7] to the problem in the transmission capacity framework, which is, to the best of our knowledge, a novel approach.

The remainder of this paper is structured as follows. In Section II the system model is introduced. Section III restates results by Jindal et al. [4] for comparison. The transmission capacity of networks with local FDMA scheduling is derived in Section IV and compared with the no scheduling case. Section V offers concluding remarks.

\section{SYSTEM MODEL}

The wireless network considered consists of nodes $\left\{X_{i}\right\}$ distributed in the plane according to a stationary Poisson point process (PPP) $\Pi$ of intensity $\lambda$, where the $X_{i} \in \mathbb{R}^{2}$ denote the locations of transmitters causing interference. The network bandwidth $B$ is the total bandwidth available for communication and is split into $M$ orthogonal channels of system bandwidth $B_{m}=\frac{B}{M}$. The system bandwidth is the high frequency bandwidth of a single node. Ambient noise power spectral density is $N_{0}$. The power attenuation factor between two points in the plane at distance $d$ is given by $d^{-\alpha}$, $\alpha>2$ (path loss model). According to Slivnyak's Theorem, cf. [8], the statistics of $\Pi$ are not affected by the addition of a reference transmitter and receiver pair in each channel. The channels are symmetric, so it suffices to consider one reference pair. The reference receiver is placed at the origin, the reference transmitter $r$ meters away. Each transmitter, both the reference transmitter and interferers, transmits with power 
$\rho$ and hence spectral power density $\frac{\rho}{B_{m}}=\frac{\rho M}{B}$ in a channel. The performance of the reference receiver depends on the interference in a channel generated by nodes operating in the same channel. As the PPP is homogeneous, the performance of the reference receiver also characterizes the performance of the whole network.

We assume that the network protocol is capable of orthogonalizing the transmissions of all neighbors around the reference receiver in an orthogonalization radius $r_{o}$. Concurrent transmissions in the same area thereby take place on different channels, resulting in a guard zone free of interfering transmitters. More channels mean less interference as more neighbors can be orthogonalized and less interference comes from all other nodes since their activity is split onto $M$ channels. On the other hand, less spectrum is available for a point-to-point link, resulting in a higher outage probability for a given data rate. In the following sections, we will quantify this trade-off and find the optimum number of channels $M$.

\section{TRANSMISSION CAPACITY WITHOUT LOCAL SCHEDULING}

First, we will derive the relationship between system bandwidth, operating bandwidth and transmission capacity under internal interference for a network which needs to support a (minimum) data rate $R_{m}$ at a (maximum) distance $r$ in each point-to-point connection without local scheduling. There is an optimal split of the operating bandwidth $M_{\mathrm{opt}}$ for which outage is minimized, independently of $\lambda$. The transmission capacity of the network is the goodput of the associated node density.

The signal to interference and noise ratio (SINR) experienced by the reference receiver in a channel $m$ can be written as

$$
\mathrm{SINR}=\frac{\rho r^{-\alpha}}{N_{0} B_{m}+\sum_{i \in \Pi_{m}\left(\lambda_{m}\right)} \rho\left|X_{i}\right|^{-\alpha}},
$$

where $\lambda_{m}$ denotes the PPP intensity in channel $m$. In channel $m$ the outage probability $q_{m}$ for a transmission rate $R_{m}$ is

$$
\begin{aligned}
q_{m}\left(\lambda_{m}\right) & =\mathbb{P}\left\{B_{m} \log _{2}(1+\mathrm{SINR}) \leq R_{m}\right\} \\
& =\mathbb{P}\{\underbrace{\mathrm{SINR}^{-1}}_{Y_{m}}>\underbrace{\frac{1}{2^{\frac{R_{m}}{B_{m}}}-1}}_{=: 1 / \beta}\} .
\end{aligned}
$$

Making use of symmetry, the two-dimensional PPP of $\Pi_{m}\left(\lambda_{m}\right)$ can be mapped to a one-dimensional PPP with unit intensity [9], [1] and $Y_{m}$ hence written as:

$$
Y_{m}=\frac{N_{0} B_{m}}{\rho r^{-\alpha}}+\left(\pi r^{2} \lambda_{m}\right)^{\frac{\alpha}{2}} \underbrace{\sum_{i \in \Pi_{1}(1)} T_{i}^{-\frac{\alpha}{2}}}_{Z_{\alpha}},
$$

where $T_{i}$ is the distance to the origin of the interferer $i$. It follows for $q_{m}\left(\lambda_{m}\right)$ :

$$
q_{m}\left(\lambda_{m}, R_{m}\right)=\mathbb{P}\left\{Z_{\alpha}>\left(\pi r^{2} \lambda_{m}\right)^{-\frac{\alpha}{2}}(\underbrace{\left.\frac{1}{2^{\frac{R_{m}}{B_{m}}}-1}-\frac{N_{0} B_{m}}{\rho r^{-\alpha}}\right)}_{\theta_{m}}\} .\right.
$$

Let $\bar{F}_{Z_{\alpha}}$ denote the complementary cumulative distribution function of $Z_{\alpha}$. Then,

$$
q_{m}\left(\lambda_{m}\right)=\bar{F}_{Z_{\alpha}}\left(\left(\pi r^{2} \lambda_{m}\right)^{-\frac{\alpha}{2}} \theta_{m}\right) .
$$

The optimal system bandwidth of each channel is given by minimizing the outage probability:

$$
M_{\mathrm{opt}}:=\underset{M}{\arg \min } q_{m}\left(\lambda_{m}, R_{m}\right)
$$

Inserting (5) and assuming a symmetric distribution of nodes ${ }^{1}$ to channels with $\lambda_{m}=\frac{\lambda}{M}$ and $B_{m}=\frac{B}{M}$ yields

$$
\begin{aligned}
M_{\mathrm{opt}} & :=\underset{M}{\arg \min }\left(\bar{F}_{Z_{\alpha}}\left(\frac{\theta_{m}}{\left(\pi r^{2} \lambda_{m}\right)^{\frac{\alpha}{2}}}\right)\right) \\
& =\underset{M}{\arg \max }\left(\frac{\theta_{m}}{\left(\pi r^{2} \lambda_{m}\right)^{\frac{\alpha}{2}}}\right) \\
& =\underset{M}{\arg \max }\left(\left(\left(\pi r^{2} \frac{\lambda}{M}\right)^{-\frac{\alpha}{2}}\left(\frac{1}{2^{\frac{R_{m}}{B} M}-1}-\frac{N_{0} B}{\rho r^{-\alpha} M}\right)\right)\right) \\
& =\underset{M}{\arg \max }\left(M^{\frac{\alpha}{2}}(\frac{1}{2^{\frac{R_{m}}{B} M}-1}-\underbrace{\frac{N_{0} B}{\rho r^{-\alpha} M}}_{N_{0} / E_{b}})\right.
\end{aligned}
$$

This optimization problem is solvable in closed form for the interference-limited regime (thermal noise is assumed negligible, $\frac{E_{b}}{N_{0}} \rightarrow \infty$ ) [4]. It follows

$$
M_{\mathrm{opt}}=\frac{B}{R_{m}} \underbrace{\log _{2} \exp \left(\frac{\alpha}{2}+\mathcal{W}\left(-\frac{\alpha}{2} \exp \left(-\frac{\alpha}{2}\right)\right)\right.}_{b_{\mathrm{opt}}})
$$

where $\mathcal{W}$ denotes the principal branch Lambert-W function. The optimal split ${ }^{2}$ hence depends on $\alpha$, desired supportable rate $R_{m}$ and operating bandwidth $B$.

This is also, under the given system model assumptions, the optimum operating bandwidth split for an FH-CDMA ad hoc network to minimize outage at a given point-to-point data rate. The split corresponds to an SINR threshold $\beta$ of

$$
\beta_{\mathrm{opt}}=2^{b_{\mathrm{opt}}}-1
$$

and a spectral efficiency in all links of $b_{\text {opt }}$.

The transmission capacity of a channel is defined as [1]

$$
c_{m}\left(q_{m}\right)=\lambda_{m}\left(q_{m}\right)\left(1-q_{m}\right)
$$

where the function $q_{m}^{-1}(\epsilon)=\lambda_{m}$ denotes the inverse of the outage probability and gives the spatial intensity $\lambda_{m}$ of intended transmissions associated with an outage probability $\epsilon$. It is multiplied by the probability of success $1-\epsilon$.

The transmission capacity is a measure of the spatial goodput and gives the number of nodes transmitting successfully in a unit area at a given time.

\footnotetext{
${ }^{1}$ The symmetric distribution to channels is justified due to the homogeneous PPP model and equal background noise in each channel.

${ }^{2}$ Assuming $B$ or $R_{m}$ are adjusted so that $M_{\mathrm{opt}}$ is an integer, otherwise the optimal $M$ will be the ceiling or floor of the expression.
} 
In a multi-channel network, the (total) transmission capacity is given by

$$
c(\epsilon)=\sum_{m=1}^{M} c_{m}(\epsilon), \epsilon \in(0,1) .
$$

Using (5), (8),

$$
c(\epsilon)=\frac{\bar{F}_{Z_{\alpha}}^{-1}(\epsilon)^{-\frac{2}{\alpha}}}{\pi r^{2}}(1-\epsilon) \beta_{\mathrm{opt}}^{-\frac{2}{\alpha}} \underbrace{\frac{B}{R_{m}} b_{\mathrm{opt}}}_{M_{\mathrm{opt}}} .
$$

The transmission capacity in (12) scales linearly with operating bandwidth. In multi-channel ad hoc networks, the ratio $\frac{R_{m}}{B}$ will typically be small, resulting in a high number of channels. In the interference-limited regime, the transmission capacity can be made arbitrarily large by extending the operating bandwidth.

In general, the transmission capacity (12) cannot be explicitly computed. A special case is $\alpha=4$, where the transmission capacity is, $Q(z)=\mathbb{P}(Z \leq z)$ for $Z \sim N(0,1)$, [1]

$$
c(\epsilon)=\frac{\sqrt{2 / \pi} Q^{-1}((1+\epsilon) / 2)}{\pi r^{2} \sqrt{\beta_{\mathrm{opt}}}}(1-\epsilon) M_{\mathrm{opt}} .
$$

This case will be used for comparison.

\section{TRANSmission CAPACiTy OF Wireless Networks WITH LOCAL FDMA SCHEDULING}

\section{A. Feasibility of Orthogonalization}

An important question to ask first is that of feasibility of orthogonalization of $N$ neighbors when $M=N+1$ channels are available. This corresponds to a graph vertex coloring problem with $M$ colors in an infinite random graph. Only if orthogonalization is feasible anywhere in the network with high probability, the reference link can be regarded as accurately describing the performance of the whole network. By Brooks' theorem [7], successful orthogonalization (coloring) of a graph with node (vertex) set $\left\{X_{i}\right\}$ is feasible with $M$ channels (colors), if the maximum number of neighbors $\max \left\{N_{i}\right\}$ (maximum degree) is less than $M$. Obviously, this can only be the case for a finite number of nodes. Hence, the PPP $\Pi$ is conditioned on having $K$ nodes on an area of interest $A \subset \mathcal{R}^{2}$, where $\frac{K}{A}=\lambda$. Given a PPP with intensity $\lambda$, the number of neighbors $N_{i}$ of each node $X_{i}$ is iid. Poisson distributed with $\lambda_{n}=\pi r_{o}^{2} \lambda$. A sufficient condition for feasibility of orthogonalization with probability greater than $1-\epsilon_{o}$ is that the maximum of the set of Poisson random variables describing the number of neighbors $N_{i}$ of each node $X_{i}$ is not greater than or equal to the number of colors available:

$$
\mathrm{P}\left\{\max \left\{N_{1}, N_{2}, \ldots, N_{K}\right\} \leq M-1\right\}>1-\epsilon_{o}
$$

By making use of the independence of the random variables, this condition yields

$$
\begin{aligned}
1-\epsilon_{o}(M) & <\left(\sum_{i=0}^{M-1} \exp \left(-\lambda_{n}\right) \frac{\lambda_{n}^{i}}{i !}\right)^{K} \\
& =\Phi\left(M, \lambda_{n}\right)^{K} .
\end{aligned}
$$

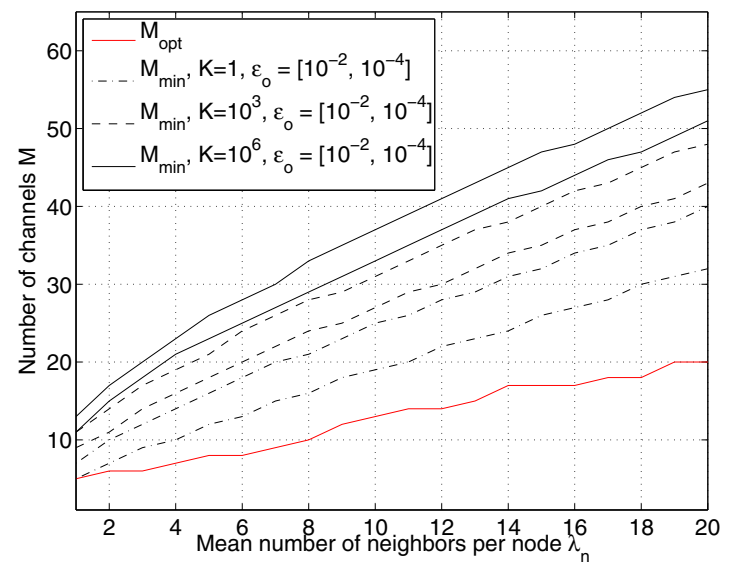

Fig. 1. Number of channels required to allow orthogonalization in a network of $K$ nodes with probability $\epsilon_{O}$ and number of channels minimizing point-topoint outage $M_{\text {opt }}$ in the local scheduling case. $M_{\mathrm{opt}}$ is determined via Monte Carlo simulations for $R_{m} / B=0.1, \lambda_{n}=5, \alpha=4$ and $r=10$.

where $\Phi(s, x)=\frac{\Gamma\left(M, \lambda_{n}\right)}{\Gamma(M)}$ is the regularized gamma function. As $M$ grows, $\epsilon_{o}(M)$ rapidly converges to 0 . In fact, denoting $\exp (x)=\sum_{i=0}^{M-1} \frac{x^{i}}{i !}+R_{M}(x), \epsilon_{o}(M)$ can be upper bounded by

$$
\begin{aligned}
\epsilon_{o}(M) & =1-\left(\sum_{i=0}^{M-1} \exp \left(-\lambda_{n}\right) \frac{\lambda_{n}^{i}}{i !}\right)^{K} \\
& =1-\left(1-\exp \left(-\lambda_{n}\right) R_{M}\left(\lambda_{n}\right)\right)^{K} \\
& \leq 1-\left(1-\exp \left(-\lambda_{n}\right) \frac{2 \lambda_{n}^{M}}{M !}\right)^{K}
\end{aligned}
$$

for $M \geq 2 \lambda_{n}-2$ (making use of $R_{M}(x) \leq 2 \frac{|x|^{M}}{M !}$, proof by bounding the remainder term of the exponential series).

Given an area $A, \lambda, \epsilon_{o}>0$ and $r_{o}$, the minimum number of channels needed for FDMA orthogonalization within radius $r_{o}$ can be obtained by solving (15) for $M$, according to

$$
M \geq \Phi^{-1}\left(\left(1-\epsilon_{o}\right)^{\frac{1}{K}}, \lambda_{n}\right) .
$$

Figure 1 shows the number of channels needed for various $K$ and $\epsilon_{o}$. Somewhat surprisingly, even for a large number of nodes $K$ and small $\epsilon_{o}$ the required minimum number of channels grows slowly. This is one of the properties of the distribution of the maximum of a set of independent Poisson random variables as further examined in [10].

\section{B. Outage and Transmission Capacity}

Let us now assume that the network uses $M$ channels and is agile to coordinate the transmission of neighboring nodes to avoid interference in such a way that it always finds an orthogonalization if it is feasible. The probability $1-\epsilon_{o}(M)$ of a feasible orthogonalization in a network with $K$ nodes and $M$ channels is given by (15). If the probability of network orthogonalization is high, a representative measure is the probability of a locally feasible orthogonalization. This is the probability that not more than $M-1$ nodes lie within the orthogonalization range of the reference node: 


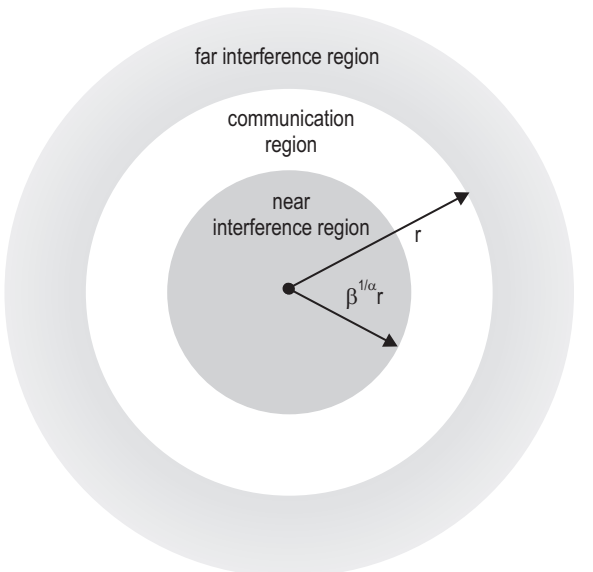

Fig. 2. $\beta \leq 1$ : The communication region is greater or equal to the near interference region.

$$
p_{\mathrm{o}}=\sum_{i=0}^{M-1} \exp \left(-\lambda_{n}\right) \frac{\lambda_{n}^{i}}{i !}
$$

For the following considerations, we assume that $M A C$ scheduling can eliminate $M-1$ near interferers in the transmission range $r$, hence $r_{o}=r$, by assigning them to different frequency channels. The assumption is reasonable: if nodes can communicate directly, they can also effectively exchange their transmission schedule.

Similar to the no scheduling case, closed form solutions of such an interference field do not exist: we will derive upper and lower bounds on the outage probability.

The outage event can be decomposed by considering the communication region, the near interference region and the far interference region. The communication region and the near interference region are discs of radius $r$ and $r_{s}=\beta^{\frac{1}{\alpha}} r$, respectively. The far interference region is the area outside the near interference region. Interfering nodes inside the near interference region directly cause outage. Interfering nodes in the far interference region can cause outage, if the aggregate interference exceeds $\frac{1}{\beta}$.

The overall trade-off to be examined can be characterized as follows: A higher number of channels increases the feasibility of orthogonalization and reduces the total number of interferers in a channel, but at the same time increases power spectral density of each interferer and reduces the usable point-to-point bandwidth, which still needs to support the same data rate.

Two cases as shown in Figure 2 and 3 have to be differentiated. For $\beta>1$, the near interference range is greater than the communication range. For $\beta \leq 1$, the communication range is greater than the near interference range. For both cases, we derive bounds on outage for a given number of channels and show the associated transmission capacity in relations to the no scheduling case.

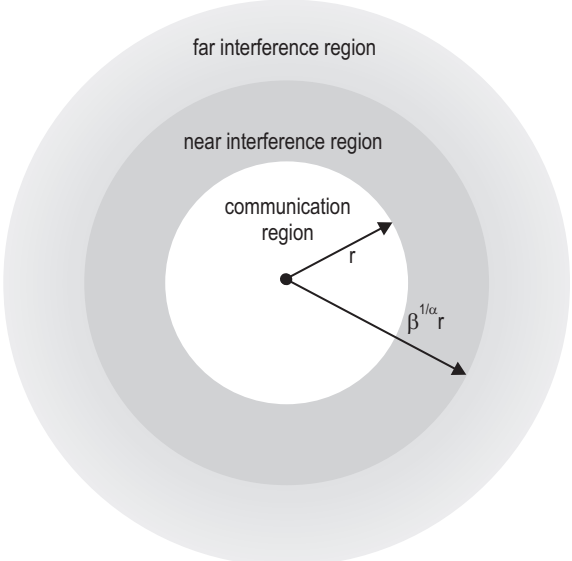

Fig. 3. $\beta>1$ : The communication region is smaller than the near interference region. The near interference region hence extends beyond the communication range.

C. Low spectral efficiency - the spreading case: $\beta \leq 1$, $\frac{R_{m}}{B} M \leq 1$

From $2^{\frac{R_{m}}{B}} M-1=\beta \leq 1$, it immediately follows $\frac{R_{m}}{B} M \leq$ 1. In this case, the communication region is greater than the near interference region. The outage probability $q(\lambda)$ can be decomposed into outage due to infeasible orthogonalization around the reference receiver and outage due to far interferers. Equivalently, for successful transmission with probability $1-$ $q(\lambda)$,

$$
\begin{aligned}
1-q(\lambda)= & p_{o}(\lambda) \mathbb{P}\left\{Y_{r}(\lambda) \leq \beta^{-1}\right\}+\left(1-p_{o}(\lambda)\right) \\
& \mathbb{P}\left\{\text { no non-orth. node within } r_{s}\right\} \mathbb{P}\left\{Y_{r_{s}}(\lambda) \leq \beta^{-1}\right\}
\end{aligned}
$$

must hold, where $Y_{r}(\lambda)$ is the amount of interference created by nodes that are at least $r$ units away from the reference receiver. Given that orthogonalization has failed at the reference receiver, $\mathbb{P}$ (no non-orth. node within $r_{s}$ ) is the probability of "no non-orthogonalized node in near interference region" and $\mathbb{P}\left\{Y_{r_{s}}(\lambda) \leq \beta^{-1}\right\}$ is the probability of "the aggregate interference from the far field starting at $r_{s}$ not exceeding $\frac{1}{\beta}$ ".

Lower Bound: If we neglect the second term in (19), we obtain a lower bound on the success probability $1-q$, according to

$$
1-q(\lambda)>p_{o}(\lambda) \mathbb{P}\left\{Y_{r}(\lambda) \leq \beta^{-1}\right\}
$$

The term $\mathbb{P}\left\{Y_{r}(\lambda) \leq \beta^{-1}\right\}$ can further be lower bounded using the Markov inequality $c \mathbb{P}\{X \geq c\} \leq \mathbb{E}\{X\}$, yielding

$$
\mathbb{P}\left\{Y_{r}(\lambda) \leq \beta^{-1}\right\} \geq 1-\frac{\lambda}{M} \frac{2 \pi r^{2}}{\alpha-2} \beta .
$$

Note that this bound is loose for small $M$. For $M \rightarrow$ 1 , we have that $p_{o}=0$ and thus (19) is replaced by $\mathbb{P}\left\{\right.$ no node within $\left.r_{s}\right\} \mathbb{P}\left\{Y_{r_{s}}(\lambda) \leq \beta^{-1}\right\}$.

Upper Bound: An upper bound on the success probability can be derived by assuming that the next $M-1$ neighbors of the reference receiver can always be orthogonalized and just 


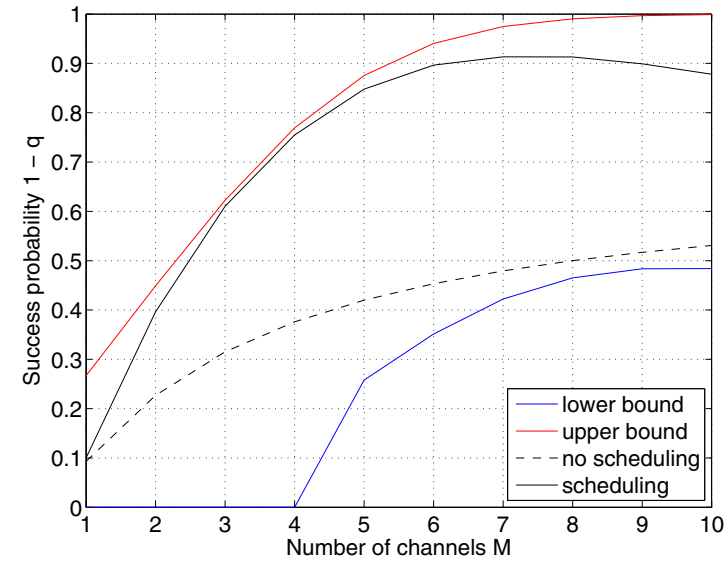

Fig. 4. Success probability for $\beta \leq 1: R_{m} / B=0.1, \lambda_{n}=5, \alpha=4$, $r=10$.

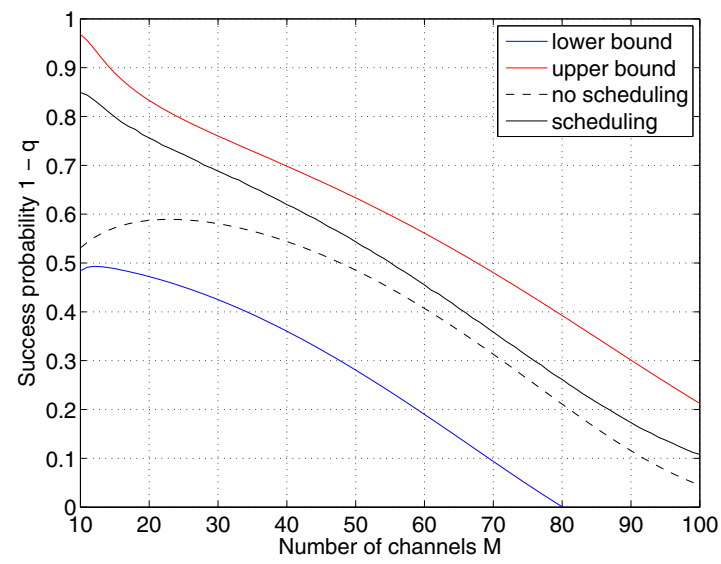

Fig. 5. Success probability for $\beta \geq 1: R_{m} / B=0.1, \lambda_{n}=5, \alpha=4$, $r=10$.

taking into account nodes in the near interference region. In this case, a transmission is successful if no more than $M-1$ nodes are found in the near interference region. $\mathbb{P}\left\{Y_{r_{s}}(\lambda) \leq\right.$ $\left.\beta^{-1}\right\}$ can hence be upper bounded by

$$
\mathbb{P}\left\{Y_{r_{s}}(\lambda) \leq \beta^{-1}\right\} \leq \sum_{i=0}^{M-1} \exp \left(-\lambda_{s}\right) \frac{\lambda_{s}^{i}}{i !}
$$

where $\lambda_{s}=\lambda \pi\left(\beta^{\frac{1}{\alpha}} r\right)^{2}$. An upper bound on the transmission capacity is given by numerically solving (22) for $\lambda$.

Figure 4 shows the outage probability computed via Monte Carlo simulation, the lower bound (20), the upper bound based on (22) as well as the exact outage probability for the no scheduling case.

\section{High spectral efficiency: $\beta>1, \frac{R_{m}}{B} M>1$}

In the high spectral efficiency regime, the interference region exceeds the communication region. In this case, outage can be due to an orthogonalization failure, to the presence of at least one node within the annulus with radii $r_{s}=\beta^{\frac{1}{\alpha}} r$ and

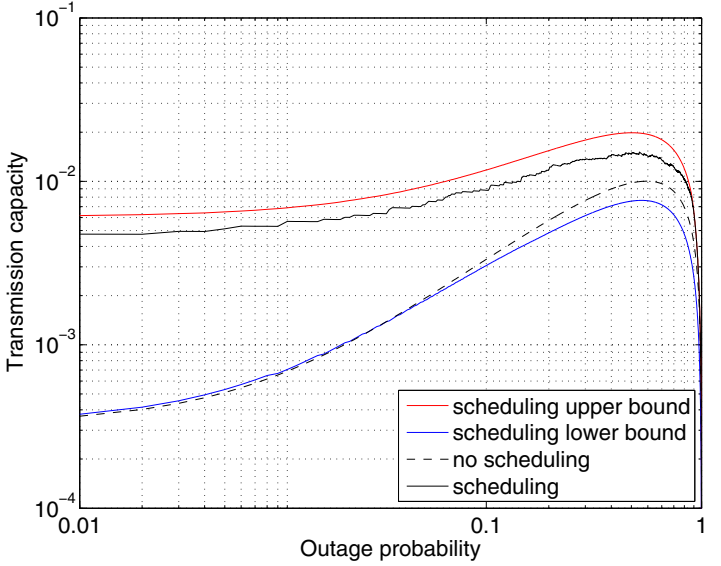

Fig. 6. Transmission capacity for $K=10^{3}, \epsilon_{o}=10^{-2}, R_{m} / B=0.1$, $\alpha=4, r=10$.

$r$, as well as to the aggregate interference from the far field exceeding $\frac{1}{\beta}$. The success probability is

$$
\begin{array}{r}
1-q(\lambda)=p_{o}(\lambda) \mathbb{P}\{\text { no node in annulus }\} \\
\qquad \mathbb{P}\left\{Y_{r_{s}}(\lambda) \leq \beta^{-1}\right\},
\end{array}
$$

where $\mathbb{P}\{$ no node in annulus $\}=e^{-\frac{\lambda}{M} \pi\left(r_{s}^{2}-r^{2}\right)}$.

Lower bound: We obtain a lower bound on $\mathbb{P}\left\{Y_{r_{s}}(\lambda) \leq\right.$ $\left.\beta^{-1}\right\}$ by using the Markov inequality, yielding

$$
1-q(\lambda) \geq p_{o}(\lambda)\left[1-\frac{\lambda}{M} \frac{2 \pi r^{2}}{\alpha-2} \beta^{\frac{2}{\alpha}}\right] e^{-\frac{\lambda}{M} \pi\left(r_{s}^{2}-r^{2}\right)} .
$$

Upper Bound: An upper bound on the transmission capacity for $\beta>1$ is obtained by assuming $p_{o}(\lambda)=1$ and neglecting far field interference. In this case, the success probability $1-q(\lambda)$ depends only on nodes situated in the annulus:

$$
1-q(\lambda) \leq e^{-\frac{\lambda}{M} \pi\left(r_{s}^{2}-r^{2}\right)}
$$

Figure 5 shows the outage probability computed via Monte Carlo simulation, the lower bound (24), the upper bound based on (25) as well as the exact outage probability for the no scheduling case.

\section{E. Discussion}

Figure 5 and 4 show that, for a fixed low number of neighbors $\lambda_{n}$ in communication range, local scheduling offers the maximum gain at a lower number of channels and hence lower spectral efficiencies. The dominating interferers are orthogonalized via FDMA and need not be avoided randomly. If high spectral efficiencies are employed, the gain of local scheduling diminishes quickly due to the fact that the dominating interferers are no longer in communication range.

Figure 6 shows the transmission capacity of local scheduling and a comparison to the no scheduling case. For the no scheduling case, the number of channels is chosen according to (7). For the scheduling case, the optimum number of channels $M_{\mathrm{opt}}$ minimizing point-to-point outage is always less 
than the number of channels required for feasible network orthogonalization, cf. Figure (1). Accordingly, the minimum number of channels for feasible network orthogonalization was chosen in Figure 6. High allowable outage probabilities lead to a high number of channels and high spectral efficiencies. An important fact to note is that the optimum number of channels minimizing link outage $M_{\text {opt }}$ now depends on the node density $\lambda$, which is not the case in the no scheduling case. The overall gain in terms of transmission capacity is a factor of 1.35 to 13, depending on the allowable outage.

\section{CONCLUSiOn AND Future WORK}

As shown in the analysis it is very beneficial to employ local FDMA scheduling in large scale dense ad hoc networks. Local FDMA scheduling yields significant gains and is less complex in implementation than, e.g., interference canceling techniques. An efficient MAC design for multi-channel ad hoc networks should hence incorporate a combination of dynamic frequency planning and adaptive link spectral efficiencies.

An interesting research direction to explore in future work is the extension of the derived results to fading channels, e.g. along the lines of existing results in [1], as well as a direct comparison of FDMA with local scheduling and successive interference cancelation.

\section{REFERENCES}

[1] S. Weber, J. Andrews, and N. Jindal, "An overview of the transmission capacity of wireless networks," IEEE Transactions on Communications, Jan 2010, under revision, http://arxiv.org/abs/0809.0016v4.

[2] S. Weber, X. Yang, J. Andrews, and G. de Veciana, "Transmission capacity of wireless ad hoc networks with outage constraints," IEEE Transactions on Information Theory, Aug 2005.

[3] J. Andrews, S. Weber, and M. Haenggi, "Ad hoc networks: To spread or not to spread," IEEE Communications, Dec 2007.

[4] N. Jindal, J. Andrews, and S. Weber, "Bandwidth partitioning in decentralized wireless networks," IEEE Transactions on Communications, Apr 2008.

[5] S. Weber, J. Andrews, X. Yang, and G. de Veciana, "Transmission capacity of wireless ad hoc networks with successive interference cancellation," IEEE Transactions on Information Theory, Aug 2007.

[6] A. Hasan and J. Andrews, "The guard zone in wireless ad hoc networks," IEEE Transactions on Wireless Communications, Mar 2007.

[7] R. Brooks, "On colouring the nodes of a network," Mathematical Proceedings of the Cambridge Philosophical Society, Apr 1941, doi: $10.1017 /$ S030500410002168X.

[8] D. Stoyan, W. Kendall, and J. Mecke, Stochastic geometry and its applications, 2nd ed. Wiley, 1995.

[9] M. Haenggi, "On distances in uniformly random networks," IEEE Transactions on Information Theory, Oct 2005.

[10] K. Briggs, L. Song, and T. Prellberg, "A note on the distribution of the maximum of a set of poisson random variables," preprint, Mar 2009, http://front.math.ucdavis.edu/0903.4373. 